\title{
Turismo alternativo: una propuesta para impulsar el desarrollo en dos localidades indígenas de Nayarit, México
}

Recibido: 16/10/2015 - Aceptado: 03/11/2015

Areli Zuleth Cueto Valdivia

Fernando Flores Vilchez*

Oyolsi Nájera González

Rigoberto Zepeda Loera

Universidad Autónoma de Nayarit

\section{Resumen}

Las localidades indígenas y sus expresiones culturales han sido consideradas desde hace varios años como un atractivo para la sociedad occidentalizada. En ese tipo de lugares, cuyas condiciones económicas suelen ser precarias, el turismo alternativo puede constituir una opción sustentable que contribuya a su mejoramiento. Con esta investigación se pretende conocer la percepción que sobre la situación actual y las perspectivas de desarrollo tiene la población de dos localidades indígenas de Nayarit y las actividades que creen factibles para impulsar tal desarrollo, entre ellas las relacionadas con el turismo alternativo. Se piensa que la diversificación de las actividades productivas que aprovechen los recursos naturales, culturales y sociales de una localidad puede ofrecer a la población autosuficiencia económica y social, aminorando la pobreza y la marginación.

Palabras clave: Nayarit, calidad de vida, desarrollo, grupos indígenas, turismo alternativo.

*Correo electrónico: vilchez@hotmail.com 


\title{
Alternative tourism: a proposal to boost development of two indigenous communities in Nayarit, Mexico
}

Areli Zuleth Cueto Valdivia

Fernando Flores Vilchez*

Oyolsi Nájera González

Rigoberto Zepeda Loera

Universidad Autónoma de Nayarit

\begin{abstract}
Indigenous communities and their cultural manifestations have been considered for the past few years as an attraction for western society. In these locations, where economic conditions are usually precarious, alternative tourism can constitute a sustainable option that contributes to their development. The objective of this research project is to recognize the perception of inhabitants of two indigenous communities in Nayarit over their present day situation and their development perspectives and to know the type of alternative tourism activities they consider viable to promote development. Diversification of productive activities that take advantage of natural, cultural and social resources of a community is considered to have the potential to offer economic and social self-sufficiency reducing poverty and marginalization.
\end{abstract}

KEY worDs: Nayarit, quality of life, development, indigenous groups, alternative tourism.

*E-mail: vilchez@hotmail.com Teoria y PraXis · ISSN 18701582 - NÚM. 20 · JULIO-DICIEMBRE 2016 · 95-123 pp. 


\section{Introducción}

La desigualdad social, los altos niveles de pobreza y el volátil crecimiento económico son considerados los problemas más profundos que históricamente ha enfrentado la región de América Latina y el Caribe (Comisión Económica para América Latina y el Caribe, 2006). Di Virgilio, Otero y Boniolo (2010) definen la pobreza como la ausencia de los ingresos necesarios para afrontar las necesidades básicas, y señalan que esta puede ser producto de la desigualdad entre extremos provocando la exclusión/inclusión diferencial, entendiendo la exclusión como una situación de marginación del disfrute del producto social; en el caso de algunos países de América Latina, no se trata de una cuestión de carencias sino de distribución, por tal motivo la pobreza y la desigualdad no solo están presentes en esta región, sino que se encuentran íntimamente ligadas. Las autoras consideran esta región como la más desigual del mundo en términos de la distribución de ingresos y de activos.

Las personas que se ven agobiadas por la pobreza y las deficiencias en materia de salud o educación, así como a las que se les niegan los derechos civiles y políticos para influir en las decisiones que las afectan, carecen de la posibilidad de elegir la vida que valoran (García, Salvatierra, Trujillo y Zúñiga, 2010). Tal condición, aunada al deterioro del empleo, facilita los procesos de concentración de capital y la diferenciación entre los niveles de vida e ingresos en estos grupos sociales; esta forma desigual de ingresos es visible en los ámbitos internacional y local (Razeto, 2001).

Las leyes y acuerdos que se han elaborado con el fin de aminorar estas diferencias sociales han reconocido los derechos de los pueblos minoritarios, sin embargo, no se ha logrado garantizar su respeto, ni las mismas posibilidades para ejercer sus derechos, lo cual, según Fernández et al. (2006), se hace patente en los grupos indígenas de México y América Latina, como uno de los sectores con altos índices de pobreza y marginación.

Dentro de las estrategias que se han implementado para revertir estas condiciones, destaca el turismo alternativo como una posibilidad para mejorar las condiciones de vida de las poblaciones locales mediante el aprovechamiento de los recursos naturales y culturales existentes. Se ha demostrado y reconocido que la actividad turística tiene la capacidad de reducir la pobreza en naciones en vías de desarrollo (Ruiz, 2008), transformando países y regiones. En la región del 
Turismo alternativo: una propuesta para impulsar el desarrollo en dos localidades

Caribe, las actividades propias del turismo alternativo en sus diversas modalidades, como el ecoturismo, generan más de $85 \%$ del producto interno bruto, presentando resultados exitosos en países como Costa Rica (Virgen, 2014), y con una tendencia creciente en el mundo.

\section{Antecedentes}

En 1983 se establece la Comisión Mundial sobre Medio Ambiente y Desarrollo, constituida por la Asamblea General de las Naciones Unidas, con el fin de ayudar a construir un futuro más próspero en el nivel mundial, para lo cual se reunirían líderes de todo el planeta y emitirían informes de sus hallazgos. En 1987, la comisión genera su primer informe, denominado "Nuestro futuro común”, conocido como Informe Brundtland, en el que se define por primera vez el término desarrollo sustentable, considerándolo como el desarrollo que satisface las necesidades del presente sin comprometer la capacidad de las generaciones futuras para que satisfagan sus propias necesidades. Por medio de este informe, la comisión exhorta a todas las naciones del orbe a que se sumen a los objetivos del desarrollo sostenible e implementen ciertas medidas para lograrlos. Algunos de los objetivos propuestos en el Informe Brundtland son: atender las necesidades humanas, asegurar niveles sustentables de población, y conservar y mejorar la base de los recursos naturales.

En 1992, cinco años después del Informe Brundtland, se llevó a cabo en Río de Janeiro la Cumbre Mundial sobre el Desarrollo Sostenible, conocida como la Cumbre de la Tierra, en la que se realizó la Cumbre de las Naciones Unidas sobre Medio Ambiente y Desarrollo, donde se enfatizó acerca de la relación que existe entre los factores medioambientales y las condiciones económicas y de justicia social (Orozco y Núñez, 2013). Cabe señalar que, durante mucho tiempo, la dimensión ambiental no fue vista como una parte importante para alcanzar el desarrollo, a menos que se tratara del medio de donde se obtenían las materias primas para la producción, es decir, era considerado solo un recurso más, disponible para satisfacer las necesidades humanas (Virgen, 2014).

En esta cumbre se logra institucionalizar el término desarrollo sostenible, pues se concretaron acuerdos y convenios que los países del mundo representados en la cumbre se comprometieron a cumplir, como los acuerdos de la Agenda 21, la Declaración de Río sobre Medio Ambiente y Desarrollo, la Declaración 
de Principios sobre Bosques, y los convenios Marco de las Naciones Unidas y el de la Biodiversidad.

Para dar seguimiento a estos acuerdos, en 2002 se realiza en Johannesburgo la Cumbre Mundial sobre el Desarrollo Sostenible, de la cual las Naciones Unidas publicaron un informe, que en su apartado 43 trata sobre promover el desarrollo sostenible del turismo, "a fin de aumentar los beneficios que las comunidades receptoras obtienen de los recursos que aporta el turismo, manteniendo al mismo tiempo la integridad cultural y ambiental de tales comunidades y aumentando la protección de las zonas ecológicamente delicadas y del patrimonio natural” (Naciones Unidas, 2002, p. 36). En este mismo apartado se reconoce que es necesario promover el desarrollo sostenible del turismo y el fomento de las capacidades que se requieren para tal propósito, a fin de contribuir al fortalecimiento de las comunidades rurales y locales, para lo cual propone la adopción de algunas medidas, entre las que se considera ayudar a las comunidades locales a administrar las visitas del turismo para obtener mayores beneficios y un mínimo de riesgos y efectos negativos sobre sus tradiciones, cultura y medio ambiente, y así promover la diversificación de las actividades económicas.

En el ámbito turístico en México, la Secretaría de Turismo (Sectur), en colaboración con la Secretaría de Medio Ambiente y Recursos Naturales (Semarnat), presentó en el año 2000 la Política y Estrategia Nacional para el Desarrollo Turístico Sustentable, con el objetivo de promover un desarrollo turístico que fomente la equidad social, la sustentabilidad natural y la rentabilidad de las inversiones, teniendo en cuenta las necesidades de las localidades anfitrionas, los inversionistas, los prestadores de servicios turísticos y los turistas, a fin de garantizar las oportunidades de desarrollo en el futuro (Sectur, 2004). Se considera que el desarrollo turístico sustentable debe ser responsabilidad de quienes desarrollan y conforman el sector turístico, haciendo copartícipe al turista de la responsabilidad de preservar los recursos naturales y el respeto a las culturas y tradiciones de las localidades receptoras (Sectur, 2004).

Uno de los paradigmas que ven al turismo como una alternativa de desarrollo sustentable es el de desarrollo local, el cual surge como una propuesta para dinamizar la economía local por medio de la diversificación de actividades que optimicen el aprovechamiento de los recursos locales, proponiendo un desarrollo de abajo hacia arriba, con base en propuestas de origen local, y es compatible con los ideales del desarrollo sustentable. 
Turismo alternativo: una propuesta para impulsar el desarrollo en dos localidades

\section{El desarrollo local, iniciativas desde abajo}

Las revalorizaciones territoriales realizadas a través de la teoría económica concluyen en la aparición en los noventa de una gama de políticas territoriales que se denominan ahora desarrollo local, el cual enfatiza la articulación de esfuerzos en los diversos actores sociales locales, para alinear los recursos exógenos y potenciar los endógenos, en una perspectiva de mejora de la calidad de vida de la población de la región (Chauca, 2011).

Según Vázquez-Barquero (2009, p. 4): "Las iniciativas de desarrollo local surgieron en los países pobres y de desarrollo tardío, con el fin de neutralizar los efectos negativos que la globalización y el ajuste productivo produjeron en el nivel de vida de la población”. Este tipo de desarrollo se relaciona estrictamente con el estado de bienestar de las poblaciones.

El desarrollo local es un proceso de crecimiento económico y de cambio estructural que conduce a una mejora del nivel de vida de la población local, en el que se pueden identificar tres dimensiones: económica, sociocultural y políticoadministrativa (Vázquez-Barquero, 1988). En este sentido cabe mencionar que estas tres dimensiones, de las que forman parte los actores locales, públicos y privados, deben estar coordinadas para lograr el desarrollo de las localidades.

Para Cotorruelo (2001), el desarrollo local constituye una estrategia territorial competitiva que se basa en el pleno aprovechamiento del potencial de utilidad endógeno, con la inserción adecuada de recursos e impulsos estratégicos exógenos; es de carácter pluridimensional e integrado y supone un proceso sistemático y sostenible a largo plazo, mediante la participación de actores locales.

Las estrategias de desarrollo local deben ser diferentes en cada caso, ya que las necesidades de las localidades varían y para cada localidad las prioridades que se deben incluir en las políticas de desarrollo son distintas (Vázquez-Barquero, 2009). Lo anterior deja entrever lo indispensable de la participación de los pobladores locales en la definición de sus necesidades, pues nadie mejor que ellos las conoce.

De acuerdo con Arocena $(1995,2002)$, para construir el concepto de desarrollo local, debe tenerse en cuenta la realidad de las sociedades y los rasgos que han incidido en sus procesos de desarrollo, ya que la realidad en materia de desarrollo contempla el pasado, el presente y el proyecto, visto desde la multidisciplinariedad, dado que la búsqueda de opciones para el desarrollo local 
de los pueblos no debe limitarse a una disciplina, a un punto de vista ni a un actor. Asimismo señala que el desarrollo local debe darse en donde convergen la necesidad de crear riqueza y la de cuidar los recursos naturales, así como la urgencia de crear empleos y la de responder a las necesidades de la población, lo cual ocurre en la escena local.

Además, debe considerarse la identidad local -es decir, reconocerse en la historia colectiva de un territorio determinado- y el potencial de las iniciativas que surgen en este ámbito, como elemento fundamental, pues son los actores locales los portadores de las mejores propuestas para capitalizar las potencialidades, y son ellos quienes buscan que los recursos se aprovechen de la mejor manera. Pero estos no son solo individuos, sino también grupos u organizaciones que desempeñan algún rol en la sociedad local, que deben ser identificados y definir su poder de acción (Arocena, 1997).

Cabe resaltar la relación que tiene el desarrollo local con la política. Gallicchio (2010) plantea que el desarrollo local debe articularse con los procesos nacionales de desarrollo y lo contempla como una estrategia más política que económica o social, pues las políticas nacionales de desarrollo pueden marcar la pauta de la utilidad y el avance que la acción local pueda tener a mediano y largo plazo; apunta que es necesario considerar de manera simultánea acciones en torno a la gobernanza multinivel, la construcción del capital social y el desarrollo económico local. O sea, no es suficiente con territorializar el desarrollo local e implementar políticas locales si no existen voluntad política y recursos en niveles superiores, además de que claramente el enfoque es de carácter multidimensional.

\section{Turismo alternativo y desarrollo en localidades indígenas}

"Las iniciativas contribuyen al desarrollo local si afectan positivamente variables relacionadas con un mejor nivel de vida de la población e incrementan la capacidad de las localidades para utilizar sus recursos en forma innovadora y para retener la riqueza que en ellas se genera” (García, González, Sánchez y Verduzco, 1998, p. 42). Entonces, el turismo alternativo, siempre y cuando cumpla con lo antes mencionado, puede ser considerado como una actividad que propicie el desarrollo local. 
Turismo alternativo: una propuesta para impulsar el desarrollo en dos localidades

El turismo implica un desplazamiento de personas, de su lugar de residencia a otro, por motivos de descanso y recreación, generando interrelaciones o intercambios de importancia social, económica y cultural (De la Torre, 1992; Castillo y Lozano, 2006). A las personas que llevan a cabo esta actividad se les llama turistas, y a la actividad por sí misma y a los recursos necesarios y las acciones realizadas para hacer posible el fin último de la actividad, que es el consumo de los productos que para el turista se ofrecen, se le denomina actividad turística, dentro de la cual se contemplan recursos, atractivos, equipamiento, servicios, etcétera.

"En países como México, la incorporación del turismo como un sector importante de la economía nacional data de los años sesenta del siglo xx y responde precisamente a esa idea de que el turismo puede llegar a ser uno de los motores de la economía de un país, pues contribuye al desarrollo económico y social de muchos lugares o regiones” (Orozco y Núñez, 2013, p. 162).

La actividad turística, producto de la sociedad moderna, llegó a ser reconocida durante la década de los sesenta principalmente por constituir un fenómeno de masas, pero a su vez fueron evidentes los problemas que se desencadenaron en consecuencia sobre la estructura social y económica de las sociedades anfitrionas y sobre la calidad ambiental (Tomio y Badel, 2009). Durante mucho tiempo el turismo fue un fenómeno de carácter masivo y estandarizado, con elementos emblemáticos característicos del turismo masivo: el sol y la playa, los que en su tiempo formaron parte del ideal de vacaciones, pero al debilitarse este modelo tradicional de turismo, el turista tiende a pensar en diferentes formas de hacer esta actividad, las cuales apuntan hacia productos turísticos diversificados y auténticos, que se desarrollen en entornos naturales. En este sentido, el turismo es un sector que sigue creciendo, aunque de una manera más moderada y controlada que en décadas anteriores; las tendencias son hacia limitar la capacidad receptiva, diversificar la oferta y la demanda, y mejorar la calidad de los servicios.

El turismo alternativo, con propuestas como el ecoturismo, el turismo rural, el de aventura, entre otras, nace en los años noventa en vinculación con lo sostenible, y se presenta como una alternativa a la explotación económica destructiva con la que se asociaba al turismo convencional. En esta misma década, la modalidad del turismo indígena comenzó a intensificarse al relacionarse con las tendencias turísticas preocupadas por la naturaleza y la diversidad cultural 
(Pereiro, 2015), vista como una forma positiva y una opción para mejorar la calidad de vida en comunidades indígenas.

Esta actividad en comunidades indígenas puede representar una manera de hacer un turismo más reflexivo, ético y educativo (Pereiro, 2015), además de promover conexiones espirituales con la naturaleza y contribuir a la disminución de la presión que se ejerce sobre los recursos naturales, e incluso fomentar el reconocimiento de la importancia de conservarlos en su estado natural como atractivo turístico y optar por su cuidado en lugar de su destrucción. El turismo alternativo busca abordar el territorio con una nueva mirada, ampliando las opciones para atraer turistas a sitios relegados de la actividad, como el caso de algunas comunidades indígenas (Bringas, González e Igor, 2004).

La Sectur (2004) considera que las actividades de turismo alternativo se dividen en tres grandes grupos: el ecoturismo, el turismo de aventura y el turismo rural, los cuales a su vez comprenden diversas actividades.

La demanda que se ha generado desde el turismo hacia los pueblos indígenas está relacionada con la creencia de que estos últimos son los preservadores de los valores humanos más auténticos, próximos a la naturaleza y al medio ambiente, que en Occidente han sido devastados (Pereiro, 2015). El turismo de corte alternativo es una forma de cómo las poblaciones locales pueden aprovechar sus recursos naturales y culturales; en el nivel nacional, este tipo de turismo se considera una opción viable para el desarrollo de algunas etnias que habitan el territorio mexicano (Bringas et al., 2004).

Sin duda, el turismo puede ser una oportunidad para el desarrollo económico de los pueblos indígenas, sin embargo, por el ambiente de marginación que regularmente se vive en estas comunidades, existe la posibilidad de que la actividad se convierta en un nuevo mecanismo de explotación y dominación neocolonial (Pereiro, 2015).

El turismo se plantea como actividad prometedora, pero es solo que al principio sus efectos no son muy notables ni su magnitud prevista; muchas veces los residentes la perciben como una forma de compartir y preservar su cultura, pero también aparecen efectos negativos sobre el carácter y las tradiciones de las comunidades locales, principalmente en las de menor tamaño (Arias, 2006).

Autores como Díaz (2010) señalan que los pobladores locales pueden llegar a experimentar una pérdida de control sobre la comunidad, sintiéndose extraños en su propia sociedad; no obstante, existen diferentes niveles de aceptación 
- Turismo alternativo: una propuesta para impulsar el desarrollo en dos localidades

de la actividad turística entre las comunidades receptoras, debido a la intensidad en el desarrollo de la actividad, la cual, mientras aumenta, se encamina hacia un modelo que agita la vida del residente, lo que se vincula a la experiencia turística. En este contexto, Virgen (2014) observa que la aceptación de la actividad se relaciona con la cantidad de visitantes que atrae, lo cual se explica por la afluencia y el perfil del turista; cuando el lugar resulta poco conocido, el número de visitantes es reducido y los turistas son aventureros, exploradores y mantienen un contacto cercano con los habitantes, situación que cambia cuando el sitio se vuelve más accesible, con mejores servicios y mayor popularidad en el mercado.

Los aspectos vinculados con los ingresos económicos que genera el turismo en las localidades en las que se inserta son las causas principales de que en ellas se presente una actitud positiva hacia él, pero no siempre se consideran los impactos que este puede tener sobre la cultura local, la dinámica de la población y las formas de vida. El turismo goza cada vez más del reconocimiento como actividad positiva para la conservación del patrimonio natural y cultural, ya que estos conforman su máximo atractivo, pero el turismo no planificado pone en peligro las condiciones físicas, las características y la integridad de este patrimonio (Barbosa, 2007).

En México, la actividad turística tradicional (de sol y playa) no ha resultado la panacea como muchos parecen pensar. Se pueden destacar los casos de Acapulco, donde se reflejan los radicales contrastes entre un mundo de lujo y comodidad y uno de pobreza y marginación (Rivera, Vázquez, Reyes, Reyes y Caretta, 2012), y Cancún, donde el desarrollo turístico implicó la descaracterización de la población de origen maya, la pérdida de su idioma, su modo de vestir y su espacio sociocultural (Pereiro, 2015).

Uno de los primeros elementos con que debe contar la población local para decidir si se implementa o no la actividad turística en su territorio es un conocimiento detallado de lo que significa el turismo, los aspectos positivos y negativos que podría tener, además de saber que los beneficios no solo son económicos; en este proceso de información y conocimiento los pobladores deben participar en el análisis y evaluación de sus necesidades de capacitación y formación, lo cual también los favorecería (Barbosa, 2007). 
Respecto a los sitios de riqueza cultural, el turismo puede ser un arma de doble filo. Los aspectos positivos que se pueden generar en estos lugares se relacionan estrictamente con la posibilidad de recuperar las tradiciones, aunque, por otra parte, las expresiones culturales suelen ser simplificadas y transformadas para el turismo, convirtiéndolas muchas veces en parodias de los verdaderos rituales; Virgen (2014) señala, por ejemplo, el turismo religioso en Cuba, en donde la difícil situación económica ha llevado a la realización de algunos ritos con extranjeros para obtener mayores ingresos, los cuales, como los bautizos, se han masificado, distorsionando y manipulando elementos culturales que deberían ser más respetados. En este sentido, el deterioro y la destrucción del patrimonio cultural no solo degradan la vida en la comunidad receptora, sino también la experiencia turística (Barbosa, 2007). Una mala planeación de la actividad y la falta de participación de los habitantes de las localidades receptoras puede llevar a la actividad al fracaso.

Por otro lado, como ya se sabe, el turismo tiende a ser altamente estacional, de modo que para aminorar el riesgo de que una localidad sea dependiente solo de esta actividad es necesario que, a la par de los proyectos turísticos, se implementen otros proyectos productivos que diversifiquen las formas de producción (Bringas et al., 2004), es decir, que las actividades propuestas se complementen para equilibrar los ingresos en las diferentes temporalidades y evitar afectaciones en este aspecto.

En la presente investigación se analiza la situación en que se encuentran las localidades de San Pedro Ixcatán y Presidio de los Reyes en materia de desarrollo, se identifican las causas de su atraso, y el potencial que pueden tener para mejorar sus condiciones de desarrollo y calidad de vida, tomando en cuenta al turismo como impulsor del desarrollo local.

\section{Metodología}

La investigación examina las condiciones de las localidades entre los años 2014 y 2015, desde un enfoque mixto, mediante el análisis de datos, la aplicación de herramientas propias de la investigación de carácter cualitativo y observación directa: entrevistas semiestructuradas a profundidad (Robles, 2011), previo registro de los temas a abordar, entre ellos potencialidades y visiones en torno a la actividad turística en la región. 
Turismo alternativo: una propuesta para impulsar el desarrollo en dos localidades indígenas de Nayarit, México

El instrumento fue aplicado a personas clave: mayores de 18 años, con conocimientos de las localidades y su historia; autoridades civiles, tradicionales y agrarias; así como directores de instituciones educativas, personas de la tercera edad, amas de casa, entre otras, con la finalidad de obtener una muestra representativa de los grupos existentes en la población. Los entrevistados fueron seleccionados a conveniencia de manera no probabilística, definiendo los perfiles de los que se deseaba conseguir información con la ayuda de un informante clave. En total se realizaron 22 entrevistas en las que se tuvieron en cuenta las dimensiones económica, sociocultural, político-administrativa y ambiental, y su relación con el desarrollo de las localidades y la región.

\section{Localidades en estudio}

Las localidades de San Pedro Ixcatán y Presidio de los Reyes (San Pedro y Presidio) forman parte de la comunidad indígena San Pedro Ixcatán, en el municipio de Ruiz, Nayarit (figuras 1 y 2). Según el Instituto Nacional de Estadística y

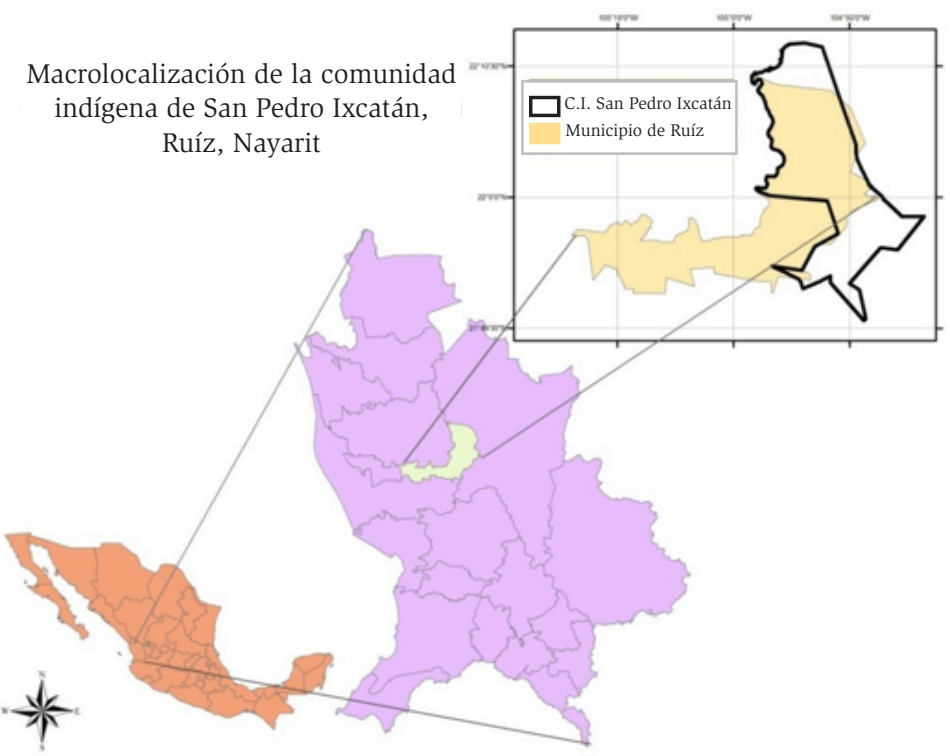

Fuente: elaboración propia

Figura 1. Microlocalización de la comunidad indígena San Pedro Ixcatán 
Geografía (INEGI, 2010), la primera cuenta con una población de 568 habitantes mestizos en su mayoría, y la segunda con 832 habitantes casi en su totalidad pertenecientes a la etnia cora (náyeri). Estas localidades se encuentran en los inicios de la zona montañosa de la Sierra Madre Occidental, al margen izquierdo del río San Pedro-Mezquital, prácticamente en el mismo espacio geográfico, separadas solo por un arroyo conocido como El Naranjo. Los estudiosos de la cultura de los coras señalan que es aquí donde se ubica el área conocida como la Región Cora Baja.
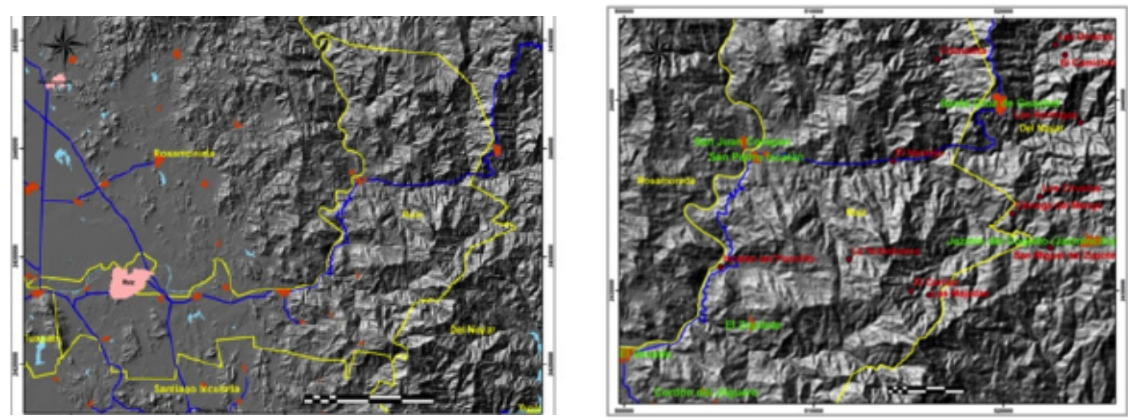

Fuente: Elaboración propia con base en INEGI 2010

Figura 2. San Pedro Ixcatán y Presidio de los Reyes

Los datos de población relevantes para las localidades en estudio, en comparación con la cabecera municipal y la ciudad de Tepic, Nayarit, presentados por el INEGi (2010), reflejan que, en el caso de Presidio, 78 \% de la población habla lengua indígena (HLI), y en el de San Pedro solamente 11 \%, lo cual da una clara idea de cómo se distribuye la población indígena en ambas localidades. En materia de educación, en Presidio solo 54 \% de la población entre 15 y 17 años asiste a la escuela, mientras que, en Tepic, el porcentaje asciende a 77.6. En Presidio, menos de la cuarta parte de la población mayor de 12 años se encuentra ocupada. Respecto a los servicios en la vivienda, entre 35 y $40 \%$ de la población de la zona en estudio no cuenta con los servicios básicos (luz eléctrica, agua entubada, drenaje), en comparación con Ruiz, donde el porcentaje es de 15 , y de Tepic, donde solamente $4 \%$ de las viviendas no tiene estos servicios. En las localidades en estudio y la cabecera municipal Ruiz, solo alrededor de $30 \%$ de la población cuenta con los servicios de salud que brindan organismos como el Instituto Mexicano del Seguro Social (Imss) y el Instituto de Seguridad 
- Turismo alternativo: una propuesta para impulsar el desarrollo en dos localidades indígenas de Nayarit, México

y Servicios Sociales de los Trabajadores del Estado (ISSSTE), mientras que en la ciudad de Tepic el porcentaje es el doble, datos que se observan en el cuadro 1.

CUADRo 1. Datos socioeconómicos de las localidades

\begin{tabular}{|c|c|c|c|c|}
\hline Localidad & $\begin{array}{l}\text { Población } \\
\text { total }\end{array}$ & $\begin{array}{l}\text { Población } \\
\text { nayor a } 3 \text { años/ } \\
\text { HLI (\%) }\end{array}$ & $\begin{array}{c}\text { Población } \\
\text { 15-17 años asiste } \\
\text { a la escuela }(\%)\end{array}$ & $\begin{array}{l}\text { Población } \\
\text { ocupada } \\
(\%)\end{array}$ \\
\hline $\begin{array}{l}\text { Presidio } \\
\text { de los } \\
\text { Reyes }\end{array}$ & 832 & 78.7 & 54.1 & 24.6 \\
\hline $\begin{array}{l}\text { San Pedro } \\
\text { Ixcatán }\end{array}$ & 568 & 11.3 & 76.6 & 32.9 \\
\hline Ruiz & 14050 & 4.4 & 68.9 & 38.0 \\
\hline Tepic & 332863 & 1.2 & 77.6 & 43.4 \\
\hline Localidad & $\begin{array}{l}\text { Total vivienda } \\
\text { habitada (Vh) }\end{array}$ & $\begin{array}{c}\text { Promedio } \\
\text { ocupantes por } \\
\text { Vh }\end{array}$ & $\begin{array}{l}\text { Vivienda sin } \\
\text { servicios } \\
(\%)\end{array}$ & $\begin{array}{c}\text { Beneficiarios de } \\
\text { servicios de salud } \\
\text { (\%) }\end{array}$ \\
\hline $\begin{array}{l}\text { Presidio } \\
\text { de los } \\
\text { Reyes }\end{array}$ & 144 & 5.78 & 36.80 & 32.9 \\
\hline $\begin{array}{l}\text { San Pedro } \\
\text { Ixcatán }\end{array}$ & 128 & 4.44 & 40.62 & 28.8 \\
\hline Ruiz & 3796 & 3.70 & 14.88 & 29.4 \\
\hline Tepic & 90733 & 3.65 & 3.45 & 60.8 \\
\hline
\end{tabular}

Fuente: Elaboración propia con base en INEGI, 2010.

\section{Resultados y discusión}

Situación económica de las localidades en estudio

Las actividades económicas que se desarrollan en la región son principalmente la agricultura de temporal y la ganadería, ambas de subsistencia, es decir, los productos obtenidos se destinan a cubrir sus necesidades de alimentación durante todo el año, primero, porque las tierras viables para cultivo son pocas y 108 se localizan en terrenos accidentados, y segundo, porque las condiciones económicas no permiten realizar inversiones significativas para una producción a 
gran escala. Según García, Salvatierra, Trujillo y Zúñiga (2010), este tipo de agricultura, con tecnología rudimentaria, determina la precariedad de las circunstancias de vida de los grupos sociales ocupados en esa actividad, aunque habría que conocer más la cultura de los grupos indígenas.

Al no ser suficiente el empleo en la región, las personas migran hacia otros sitios en busca de alternativas. En las localidades se identificaron dos modalidades de migración por empleo; la primera la representan las actividades agrícolas de la región costa del estado, como el corte de tabaco, la cosecha de frutas, verduras y legumbres, y los servicios; en este esquema, los jefes de familia se desplazan a lugares relativamente cercanos por periodos cortos y regresan cada semana a sus localidades. Sandoval (2008) sostiene que este tipo de migración es una de las estrategias de sobrevivencia que las unidades domésticas despliegan en los contextos de pobreza, pues se considera que el trabajo es el recurso más importante de los hogares pobres.

La segunda modalidad la constituye el trabajo en otros países, particularmente en Canadá y Estados Unidos de América, donde las personas se emplean en diferentes actividades. Esta opción implica temporadas de hasta seis meses fuera de sus lugares de residencia, enviando periódicamente dinero a sus familias. De acuerdo con Juárez (2015), esta migración por empleo se da en los lugares pobres donde no es posible generar los trabajos necesarios y con un ingreso adecuado para su población.

La principal fuente de ingresos en la región, después del sueldo, son los programas gubernamentales, los cuales tienen presencia todo el año mediante apoyos para estudiantes, adultos mayores, mejoramiento de vivienda, e incluso algunos fungen como fuentes de empleo, como el Programa Empleo Temporal, de la Secretaría de Comunicaciones y Transportes, para realizar limpieza y mantenimiento de las carreteras, aunque aun así el trabajo no es constante. Cabe apuntar que estos programas han generado cierto grado de dependencia en la región, y que debido a que la política asistencialista se encuentra en constante cambio, la economía de estos pueblos carece de estabilidad. Al respecto se piensa que el turismo alternativo puede mejorar este panorama, ya que, como señalan Bringas, González e Igor (2004), esta actividad se considera un factor de desarrollo local sustentable por constituir una fuente alternativa de ingresos. 
Turismo alternativo: una propuesta para impulsar el desarrollo en dos localidades

La resistencia a la occidentalización y sus manifestaciones en el área de estudio

La necesidad de salir de sus comunidades hacia otros espacios y la presencia de los programas de asistencia social han dado la apertura para que influencias externas a la población se hagan presentes originando algunos cambios en su dinámica social. Normalmente quienes migran, regresan con conductas y costumbres adoptadas en los lugares de estancia, y quienes se quedan, por lo regular se adaptan a los lineamientos y condiciones que los programas gubernamentales y la política pública traen consigo.

Esto no es nuevo. Desde que se creó el Estado-nación, los intereses del grupo dominante (mestizos) han prevalecido sobre lo que debería ser la nación, es decir, se definió que el español debería ser la lengua que se hablara en el territorio, la religión debería ser la católica y el derecho romano (Rosas, 2007); desde entonces, los indígenas no han sido vistos de forma incluyente, sino al contrario, su diferencia cultural y lingüística ha sido la causa principal de su exclusión y marginación.

Históricamente, los pueblos indígenas han luchado por mantener sus tradiciones. Un ejemplo de esto en las localidades de estudio son la separación del pueblo cora del mestizo y recientemente la implementación de una preparatoria intercultural indígena. Al respecto, Lévi-Strauss (1964) plantea que los grupos indígenas ven el mundo diferente, poseen un conocimiento concreto de su saber y lo oponen al de los mestizos. Cierto es que cada grupo y cada pueblo tiene su propio espíritu, su propia cultura, y lucha por conservarlos o protegerlos de las “amenazas” provenientes del exterior. La razón de ello es que lo que está en juego en esa lucha no es solo la identidad individual, sino la identidad colectiva (González, 2007).

Las formas de resistencia a la conquista y el sometimiento de la cultura y los modos de vida de las poblaciones indígenas se ven reflejadas en las localidades de estudio. Particularmente Presidio se funda a partir de acontecimientos de marginación y desplazamiento que sufrieron los pobladores nativos (por el año de 1951) por parte de pobladores mestizos con los que cohabitaban en la localidad de San Pedro, donde los mestizos no respetaban las tradiciones y formas de vida de los coras, por lo que los discriminaban y maltrataban hasta que estos decidieron irse del lugar y fundar su propio pueblo al otro lado del arroyo, que actualmente los divide. 
Este antecedente histórico de desplazamiento y separación por diferencias ideológicas y culturales ha traído repercusiones hasta hoy, pues los habitantes no comparten la misma visión ni intereses y aún existen resentimientos entre los pobladores. A pesar de lo anterior, no se pueden concebir las localidades por separado, ya que están relacionadas de tres diferentes maneras: primero, por el simple hecho de que es necesario pasar por San Pedro para llegar a Presidio, pues el acceso principal para ambas localidades es el mismo; segundo, porque cuentan con un nexo comercial y de empleos, y tercero, porque San Pedro es la cabecera ejidal.

Las preocupaciones de las personas de la tercera edad de la localidad se centran en la disminución del número de jóvenes que hablan la lengua materna, participan en las festividades y utilizan la vestimenta tradicional, lo que asocian con la cercanía del pueblo mestizo y la falta de respeto a sus tradiciones; de modo que a pesar de que los jóvenes toman parte en las festividades, no todos conocen su significado, solo participan por diversión y no les representa un lazo de espiritualidad con las celebraciones.

Debido a lo anterior, se propuso la creación de una escuela preparatoria intercultural (Muxatena), la cual es considerada una escuela privada, ya que funciona por cooperación, acción que manifiesta otra forma de resistencia del pueblo cora hacia la occidentalización. Cabe señalar que, en la región, esta es la institución de nivel medio superior con mayor cantidad de alumnos matriculados (74), ya que van jóvenes de otras localidades cercanas pertenecientes también a algún grupo étnico.

Esta resistencia de los coras a que sus hijos asistan a la escuela de nivel medio superior que se encuentra en San Pedro se debe a que temen que estos sean discriminados por su origen indígena, y porque consideran que en las escuelas formadas y dirigidas por mestizos no se enseña a los jóvenes a respetar la cultura y las tradiciones, lo que demuestra el poco interés que tienen por preservar las formas de vida de los grupos originarios, sus creencias y su dialecto.

Si bien los coras a lo largo del tiempo han defendido su territorio y sus costumbres, actualmente la modernización y la globalización los han alcanzado. Las vías de acceso y comunicación con el exterior son cada vez más numerosas, así como los habitantes y sus necesidades en materia de servicios, educación y empleo. Este aumento de necesidades y la creciente demanda de satisfactores, 
Turismo alternativo: una propuesta para impulsar el desarrollo en dos localidades

principalmente de las nuevas generaciones, ha llevado a la población local a reconocer la importancia de diversificar sus actividades productivas y a valorar sus recursos naturales y culturales como una posible fuente de empleo e ingresos por medio de acciones como el turismo alternativo. No obstante, se debe tener en cuenta que tratar con culturas indígenas implica arraigo cultural en lugares económicamente vulnerables, por lo que la implementación de proyectos turísticos debe contemplar la fragilidad del espacio y la aceptación de los pobladores hacia la actividad.

La visión del turismo en las localidades de estudio

Las culturas indígenas y su dinámica han sido reconocidas como un atractivo turístico, ya que se ha determinado que en estas se encuentran las raíces de los pueblos; además, sus creencias y rituales místicos resultan interesantes y atractivos para quienes desean conocer la historia de los pueblos ancestrales y sus ritos originales. Sin embargo, el turismo no siempre es respetuoso con las condiciones que se presentan en los pueblos, por lo cual se precisa que los pobladores locales estén capacitados y valoren su modo de vida indígena. La interculturalidad constituye uno de los problemas más comunes que trae consigo el turismo, ya que las influencias externas muchas veces permean los estilos de vida locales.

Actualmente, con los jóvenes en las localidades de estudio ha llegado una nueva forma de pensar, incluso son ellos quienes ven en el turismo una posibilidad de mejorar sus condiciones de vida, aunque cabe apuntar también que esta opinión no la comparten los mayores ni los más tradicionalistas, pues no muestran apertura para que personas externas al pueblo lleguen a conocer su modo de vida.

Existen en la localidad dos visiones de cómo podría darse el desarrollo turístico de la región. La primera se refiere a que algunos habitantes consideran que cuentan con los recursos naturales y culturales suficientes para ser un lugar atractivo para el turismo, y que solo hace falta organización, identificar y definir las actividades a realizar, y recibir capacitación en servicios turísticos.

La otra visión se basa en la posible construcción de una presa hidroeléctrica y su influencia en las localidades. Esta opción se relaciona estrictamente con la promesa de los beneficios que la obra podría traerles, según el discurso

$$
\text { con la promesa de los beneficios que la obra podría traerles, según el discurso }
$$


político y de los promotores del proyecto, y es aceptada mayoritariamente por la población mestiza de la región. Los siguientes párrafos pretenden ampliar la perspectiva recabada respecto a estos dos enfoques.

\section{La visión de los recursos naturales y culturales existentes como atractivo turístico}

Los pobladores locales reconocen distintos sitios naturales en la región que pueden ser atractivos para el turismo, como los manantiales de aguas termales, los balnearios naturales en los ríos y arroyos cercanos compuestos por piletas y algunas caídas de agua, las zonas de bosque (pino-encino) en los que se puede realizar la observación de algunas especies de animales y plantas, y los paisajes que se forman a lo largo de la cuenca del río San Pedro-Mezquital. Estos solo constituyen sitios de recreación para los habitantes del lugar.

Existen también atractivos representados en las expresiones culturales de los coras; su vestimenta, dialecto y formas de vida tradicionales; las artesanías, como los bolsos elaborados de lana e hilos de colores, y las prendas tradicionales del grupo étnico. No obstante que la región cuenta con influencia externa y la cercanía de lugares con formas de vida occidentalizada, y que no todos los pobladores hablan el dialecto o visten de la manera ancestral, las expresiones culturales propias de los coras se encuentran presentes en la región.
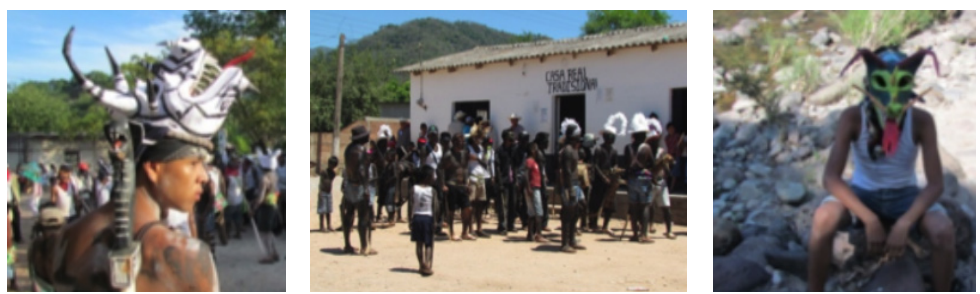

Fuente: Propiedad del autor principal.

FiguRa 3. Imágenes de la Semana Santa Cora, 2015 
Turismo alternativo: una propuesta para impulsar el desarrollo en dos localidades

En la localidad de Presidio aún se cuenta con una forma de gobierno tradicional que opera a la par del gobierno civil, pero que en los aspectos culturales es la autoridad principal, y, entre otras de sus funciones, son los encargados de las festividades tradicionales.

Una de las celebraciones más reconocidas es la Semana Santa cora (figura 3), la cual constituye uno de los principales atractivos turísticos culturales de la región. Las fechas en que esta se realiza coinciden con las de la religión católica.

A pesar de que en las localidades de estudio las festividades religiosas son similares y coinciden en fecha y creencias, en cada pueblo conservan características propias, de modo que, aparentemente, la festividad es la misma, pero los significados distintos. El origen de esto se remonta a los tiempos de evangelización de los coras por los grupos jesuitas durante la Conquista. En la actualidad se puede observar en la dinámica de la cultura religiosa de los coras una combinación del catolicismo y prácticas religiosas prehispánicas, aunque de manera general, y como lo señalan Fernández et al. (2006), solo se cuenta con una visión parcial de lo que son los pueblos indígenas, pues realmente se sabe poco sobre lo complejo de sus características, problemáticas y dinámicas, e incluso en los niveles cultural y lingüístico no se tiene completo conocimiento de los rasgos que los distinguen.

El río representa un templo para las culturas cora y huichol, ya que en él se encuentran sitios sagrados y centros ceremoniales de gran significado para ellas. El centro ceremonial de mayor relevancia en Presidio es el denominado La Muxatena, cuya importancia reúne culturalmente a habitantes de los estados de Nayarit, Durango y Jalisco, primordialmente. En este sitio se realiza también la festividad del Día de San Juan (San Juanito) el 24 de junio (figura 4), la cual es compartida entre los habitantes de Presidio y San Juan Corapan; en esta celebración se efectúa un intercambio cultural entre ambas localidades, donde el río San Pedro y el santo patrono son los actores principales. Cada año, pobladores de distintas localidades pertenecientes a diferentes grupos étnicos parten en peregrinaciones para darse cita en ese lugar, donde a pesar del clima y la fuerza del río, cruzan a san Juanito para llevarlo de un pueblo a otro y bañarse junto con él. 

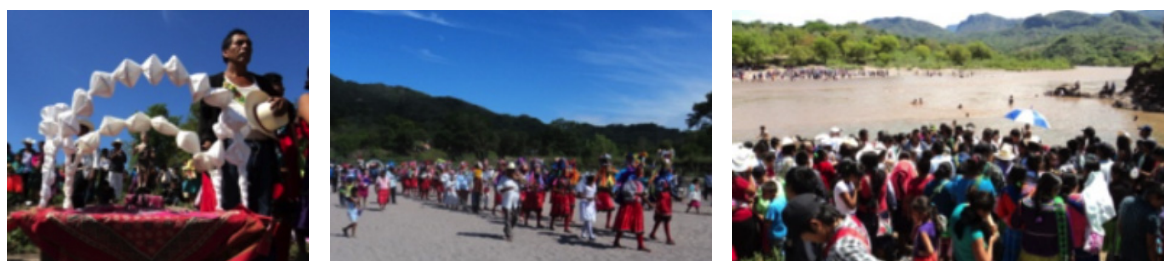

Fuente: Propiedad del autor principal.

FiguRa 4. Fiesta de San Juanito, 2015

Otras celebraciones en la región son la de Las Pachitas, que se realiza en vísperas del carnaval; el cambio de varas; la fiesta del Día de San Pedro, que es el patrono del pueblo; algunas relacionadas con la religión católica, como el Día de la Virgen y Navidad, además de la fiesta del comisariado de bienes comunales.

Cabe mencionar que algunas de las festividades tradicionales de las localidades en estudio son ya visitadas por turistas, sobre todo Semana Santa y el Día de San Juan. A pesar de que es prácticamente nula la publicidad que se les hace a estos eventos, las fechas son reconocidas por las personas que cuentan con información sobre algunas de las expresiones culturales del grupo náyeri (cora).

Varios pobladores comparten la visión de que los recursos naturales y culturales existentes son suficientes para promover la localidad como un sitio de interés turístico y con ello dinamizar su economía, y reconocen las ventajas y desventajas de implementar la actividad (cuadro 2).

\section{CuAdRo 2. Ventajas y desventajas del turismo alternativo}

\section{Ventajas}

- Encontrarse en las orillas de la carretera Ruiz-Zacatecas, la cual es la principal línea de acceso a la zona serrana de Nayarit.

- La cercanía con otras localidades que pueden ser atractivo, por contar con zonas de vegetación en buen estado

\section{Desventajas}

- No se cuenta con la infraestructura necesaria para la prestación de servicios de alimentación y hospedaje, aunque el concepto de turismo alternativo (indígena), se apega a las condiciones tradicionales y formas de vida de la localidad.

- Fragilidad sociocultural: De no ser manejada correctamente la afluencia de turistas a la zona podrían influir sobre 
Turismo alternativo: una propuesta para impulsar el desarrollo en dos localidades

CUAdRo 2. Ventajas y desventajas del turismo alternativo

(finaliza)

Ventajas

- de conservación, manantiales, ríos, así como la presencia de otras etnias como los Huicholes (en la localidad de El Naranjo).

- Que aún se cuenta con la presencia de celebraciones tradicionales de importancia para la etnia, como la Semana Santa Cora.

Fuente: Elaboración propia con base en entrevistas.

\section{La visión de la presa hidroeléctrica como detonante de la actividad turística en la región}

En Nayarit, el proyecto de mayor impacto no solo económico, sino también social, contemplado entre las propuestas del plan de desarrollo estatal para mejorar las condiciones de vida de la población es la construcción de una presa hidroeléctrica, denominada "Las Cruces", proyectada en el cauce del río San Pedro-Mezquital, muy cerca de donde se realizó la presente investigación, y en donde ya se han iniciado exploraciones y estudios previos a su construcción.

Se trata de una obra promovida por la Comisión Federal de Electriciad (CFE), la cual, según la Manifestación de Impacto Ambiental (MIA), se desarrollará en una superficie de 5349.8 hectáreas, y se localizará sobre el río San Pedro en la confluencia con el arroyo Rancho Viejo, a siete kilómetros aguas arriba de su confluencia con el arroyo El Naranjo, en el estado de Nayarit, en los municipios de Rosamorada, Ruiz, y Acaponeta. El proyecto tiene influencia sobre las localidades de San Pedro Ixcatán, Presidio de los Reyes, San Juan Corapan y San Blasito (cFe, 2013, p. 4). El objetivo de esta obra es "contribuir a satisfacer la demanda de energía eléctrica pronosticada para el área Occidente” (CFE, 2013, p. 10).

En materia de turismo, este documento reconoce que "la correcta planeación del aprovechamiento de los embalses puede contribuir a la diversificación de actividades productivas sustentables, destacando la acuacultura, turismo ecológico, turismo rural, turismo de aventura, servicios ambientales relacionados a la conservación forestal, navegación, entre otros” (CFE, 2013, p. 29), 
e incluso entre sus estrategias ecológicas dirigidas a lograr la sustentabilidad ambiental del territorio contempla rediseñar los instrumentos de política hacia el fomento productivo del turismo.

Estas visiones y propuestas han sido similares en los documentos presentados para la construcción de otras presas hidroeléctricas, ya que se considera que con las zonas de inundación se crean posibilidades de pesca, navegación, transporte y apreciación del paisaje, que pueden ser de atractivo turístico y por lo tanto detonar el desarrollo de esta actividad.

Existen antecedentes de presas hidroeléctricas que han propiciado el surgimiento de destinos turísticos importantes en México, como la denominada Miguel Alemán, en Valle de Bravo, concluida por el año de 1947 (Sierra, Romero y Zizumbo, 2012), un destino turístico reconocido como pueblo mágico, que, según datos de este programa, en 2013 tuvo una afluencia turística de 2104707 visitantes, mayoritariamente nacionales. Cabe señalar que, en Nayarit, hay tres presas hidroeléctricas: Aguamilpa, El Cajón y La Yesca, de las cuales solo en Aguamilpa se ha implementado un proyecto de turismo alternativo, en la localidad de Potrero de la Palmita, un pueblo wixárica (huichol) en el que se acondicionaron cabañas, comedores y un centro de venta de artesanías locales, además de ofrecer la participación en rituales y vivencias místicas propias del grupo étnico, pero a pesar de que han sido cuantiosos los recursos que por parte del gobierno se han invertido en ese proyecto, no se ha tenido el despegue y los resultados esperados, pues el destino no ha obtenido un reconocimiento significativo fuera de la región.

En torno a la construcción del proyecto Las Cruces existen diferentes opiniones entre los pobladores locales. A decir de los simpatizantes, esta puede ser una gran oportunidad de desarrollo para las localidades que se encuentran a sus alrededores; entre los principales aspectos positivos percibidos por estas personas están la generación de empleos, la llegada de mejores servicios e infraestructura, y el fomento de la actividad turística en la zona. Por otro lado, quienes no simpatizan con el proyecto prevén que la obra traerá impactos negativos, como la pérdida de sitios culturales de importancia y zonas de cultivo, y el acceso al río, que es una fuente directa de alimentos y recreación.

Estas opiniones opuestas, de alguna forma reflejan confusión y falta de información sobre el proyecto, y se resumen en la creencia de que los pobladores locales serán jurídica y socialmente afectados, pero económicamente beneficiados, 
Turismo alternativo: una propuesta para impulsar el desarrollo en dos localidades

por lo menos mientras duren los empleos que les han prometido, por un periodo aproximado de cinco años.

Si bien estas dos visiones tienen diferencias en cuanto a la concepción de los recursos y su aprovechamiento turístico, ambas coinciden en reconocer al turismo alternativo como una opción de diversificación económica. Las festividades tradicionales y los recursos naturales con potencial turístico se encuentran bien definidos, sin embargo, se advierte que la principal limitación para implementar la actividad es la separación de ideologías que existe entre los pobladores de la zona respecto a la forma de aprovechar los recursos disponibles, así como la resistencia hacia el turismo por parte de algunos sectores de la población.

Los resultados obtenidos en este trabajo permiten establecer que Presidio y San Pedro cuentan con las características que, de acuerdo con Arocena (1995, 1997, 2002), son necesarias para propiciar el desarrollo local: la presencia de un grupo social que sea local, es decir, que esté instalado en un territorio, que tenga características específicas que se reflejen en su cultura e identidad -como las presentes en el pueblo cora-, y que entre los individuos y actores que lo componen exista la visión y la participación para construir un espacio donde las condiciones de vida sean mejores para todos. En este sentido, la perspectiva hacia la posibilidad de implementar el turismo alternativo en la zona en estudio es bien vista.

\section{Conclusiones}

Con la demanda cada vez más diversificada de los productos turísticos, el turismo es sin duda percibido como una fuente de oportunidades con posibilidades de instaurarse en todos los rincones del planeta, pero que esta constituya una actividad transformadora no implica necesariamente que sea positiva o desarrolladora; los cambios que genera el turismo a largo plazo ocasionan casi siempre efectos negativos en la cotidianidad y realidad de las localidades receptoras, los cuales se manifiestan en sus condiciones económicas, ambientales y culturales.

- La falta de oportunidades y empleo que se presenta en la región hace necesaria la búsqueda de alternativas para aminorar la migración y los problemas sociales en las localidades de estudio; desde esta perspectiva, el turismo alternativo podría representar una fuente de ingresos y un 
factor de desarrollo en las localidades de San Pedro Ixcatán y Presidio de los Reyes.

- La población local identifica los recursos naturales y culturales con potencial de atractivo turístico y reconoce en el turismo alternativo una actividad viable de desarrollarse en sus localidades.

- Las localidades en estudio cuentan con recursos naturales y culturales de atractivo turístico que son reconocidos por los habitantes locales; para su aprovechamiento se propone la realización de actividades turísticas de bajo impacto propias del turismo alternativo, como senderismo, avistamiento de especies, safaris fotográficos, paseos a caballo, implementación de centros de interpretación y educación ambiental, uso y manejo tradicional de plantas medicinales, reconocimiento de especies de flora y fauna de la zona, elaboración y venta de artesanías (morrales, vestimenta, huaraches, indumentaria, bisutería), entre otras.

- Los pobladores consideran que la construcción de la presa hidroeléctrica podría traer oportunidades de empleo y la diversificación de sus actividades productivas por medio del turismo. No obstante, es necesario definir las condiciones bajo las que se darán estos cambios y establecer acuerdos que beneficien a la población local.

Es necesario tener en cuenta que la implementación del turismo en cualquier espacio tiene implicaciones positivas y negativas, particularmente en lugares con presencia de población indígena, en los que la transculturación y la pérdida de identidad podrían afectar la dinámica local, por lo cual es menester analizar la fragilidad de estos espacios e identificar mecanismos que permitan aminorar los impactos negativos, como la concientización y sensibilización sobre la importancia de preservar la cultura y las tradiciones, y la capacitación de la población en torno a las demandas de la actividad turística.

\section{Fuentes consultadas}

Arias, C. (2006). Enfoques teóricos sobre la percepción que tienen las personas. Horizontes Pedagógicos, 1(8), 9-22. Recuperado de http://ibero-revistas. metabiblioteca.org/index.php/rhpedagogicos/article/view/590/549 [2015, 5 de mayo]. 
- Turismo alternativo: una propuesta para impulsar el desarrollo en dos localidades

Arocena, J. (1995). El desarrollo local. Un desafío contemporáneo. Recuperado de http://es.scribd.com/doc/97660513/AROCENA-DesarrolloLocal [2015, 2 de marzo].

Arocena, J. (1997). Globalización, integración y desarrollo local. Apuntes para la elaboración de un marco conceptual. Recuperado de http://www. cedet.edu.ar/Archivos/Bibliotecas/arocena.pdf [2015, 2 de marzo].

Arocena, J. (2002). El desarrollo local: un desafío contemporáneo (2a ed.). Uruguay: Taurus/Universidad Católica. Recuperado de http://fundacionparticipar.org.ar/biblioteca/Arocena_\%20Eldesarrrollolocalundesafiocontemporaneo.pdf [2015, 3 de mayo].

Barbosa, M. (2007). La política del turismo cultural y el diseño de producto turístico para el turismo cultural. Caso: corredor turístico Bogotá-Boyacá-Santander. Escuela de Administración de Negocios, 60, 105-122. Recuperado de http://journal.ean.edu.co/index.php/Revista/article/ view/407/401 [2015, 10 de agosto].

Bringas, N., González, A. e Igor, I. (2004). El turismo alternativo: una opción para el desarrollo local en dos comunidades indígenas de Baja California. Economía, Sociedad y Territorio, 4(15), 551-590. Recuperado de http://www.redalyc.org/articulo.oa?id = 11101508 [2015, 3 de septiembre].

Castillo, M. y Lozano, M. (2006). Apuntes para la investigación turística. Quintana Roo, México: Universidad de Quintana Roo.

Chauca, P. (2011). Desarrollo regional y desarrollo local: matices y contrastes teóricos. Ideas CONCYTEG, 6(77), 1307-1329. Recuperado de http:// concyteg.gob.mx/ideasConcyteg/Archivos/77_3_CHAUCA.pdf [2015, 3 de abrill.

Comisión Económica para América Latina y el Caribe. (2006). La protección social de cara al futuro: acceso, financiamiento y solidaridad. Montevideo: Autor. Recuperado de http://www.cepal.org/publicaciones/ xml/9/24079/lcg2294e.pdf [2015, 22 de junio].

Comisión Federal de Electricidad (CFE). (2013). Manifestación de Impacto Ambiental, proyecto hidroeléctrico Las Cruces. Autor. 
Cotorruelo, R. (2001). Aspectos estratégicos del desarrollo local. En O. Madoery y A. Vázquez Barquero (eds.), Transformaciones globales, instituciones y políticas de desarrollo local. Rosario: Homo Sapiens. Recuperado de http://www.ecpunr.com.ar/Docs/bc5210d8daf1c99_cotorruelo.pdf [2015, 26 de junio].

Di Virgilio, M. M., Otero, M. P. y Boniolo, P. (coords.). (2010). Pobreza y desigualdad en América Latina y el Caribe. Buenos Aires: Consejo Latinoamericano de Ciencias Sociales. Recuperado de http://www.crop.org/ viewfile.aspx?id $=278$ [2015, 26 de agosto].

Díaz, R. (2010). La actitud del residente en el destino turístico de Tenerife: evaluación y tendencia. PASOS. Revista de Turismo y Patrimonio Cultural, 8(4), 431-444.

Fernández, P., Tuirán, A., Ordorica, M., Salas, G., Camarena, R. y Serrano, E. (2006). Informe sobre desarrollo humano de los pueblos indígenas de México 2006. México: Comisión Nacional para el Desarrollo de los Pueblos Indígenas/Programa de las Naciones Unidas para el Desarrollo. Recuperado de http://www.cdi.gob.mx/idh/informe_desarrollo_humano_pueblos_indigenas_mexico_2006.pdf [2015, 2 de octubre].

Gallicchio, E. (2010). El desarrollo local: iterritorializar políticas o generar políticas territoriales? Reflexiones desde la práctica. Eutopía, 1, 11-23.

García, M., González, S., Sánchez, A. y Verduzco, B. (1998). Descentralización e iniciativas locales de desarrollo. Guadalajara, México: Universidad Autónoma de Guadalajara.

García, N., Salvatierra, B., Trujillo, L. y Zúñiga, M. (2010). Mortalidad infantil, pobreza y marginación en indígenas de los altos de Chiapas, México. Ra Ximhai, 6(1), 115-130. Recuperado de http://www.uaim.edu.mx/ webraximhai/Ej16articulosPDF/14Mortalidad\%20Infantil.pdf [2015, 20 de marzo].

González, L. (2007). Implicaciones culturales de la globalización. Estudios Centroamericanos, 62(703-704), 377-380. Recuperado de http://www.uca. edu.sv/publica/ued/eca-proceso/ecas_anter/eca/2007/703-704/art2eca \%20703-704.pdf [2015, $1^{\circ}$ de septiembre].

Instituto Nacional de Estadística y Geografía (INEGI). (2010). Censo Nacional de Población y Vivienda 2010. México: Autor. 
- Turismo alternativo: una propuesta para impulsar el desarrollo en dos localidades

Juárez, J. (2015). Migración indígena hacia espacios agrícolas marginados de México. Un caso para contar. Agricultura, Sociedad y Desarrollo, 12(1), 87-105. Recuperado de http://www.redalyc.org/articulo. oa? $\mathrm{id}=360538155005$ [2015, 3 de septiembre].

Lévi-Strauss, C. (1964). El pensamiento salvaje. México: Fondo de Cultura Económica.

Naciones Unidas. (2002). Informe de la Cumbre Mundial sobre el Desarrollo Sostenible Johannesburgo (Sudáfrica). Nueva York: Autor. Recuperado de http://www.cepal.org/rio20/noticias/paginas/6/43766/WSSD_Informe.ESP.pdf [2015, 13 de agosto].

Orozco, J. y Núñez, P. (2013). Las teorías del desarrollo. En el análisis del turismo sustentable. InterSedes: Revista de las Sedes Regionales, 14(27), 144167. Recuperado de http://redalyc.org/articulo.oa?id $=66627452008$

Pereiro, X. (2015). Reflexión antropológica sobre el turismo indígena. Desacatos, $47,18-35$.

Razeto, L. (2001). Desarrollo económico y economía de solidaridad: El desarrollo como expansión, transformación y perfeccionamiento de la economía en el tiempo. Polis 1(1), 1-24. Recuperado de http://www.redalyc. org/articulo.oa?id = 30501117 [2015, 11 de agosto] .

Rivera, J., Vázquez, V., Reyes, H., Reyes, O. y Caretta, M. (2012). Potencialidades y desafíos del turismo y ecoturismo en el estado de San Luis Potosí, México: retos y expectativas para alcanzar el desarrollo regional. Pasos, 10(3), 289-301. Recuperado de http://www.pasosonline.org/Publicados/10312/PASOS29.pdf [2015, 5 de mayo].

Robles, B. (2011). La entrevista en profundidad: una técnica útil dentro del campo antropofísico. Cuicuilco, 18(52), 39-49. Recuperado de http://www. redalyc.org/pdf/351/35124304004.pdf [2015, 6 de julio].

Rosas, R. (2007). Exclusión, marginación y desarrollo de los pueblos indígenas. Ra Ximhai, 3(3), 693-705. Recuperado de http://www.redalyc.org/articulo.oa?id = 46130304 [2015, 10 de agosto].

Ruiz, O. (2008). Turismo: Factor de desarrollo y competitividad en México. Documentos de Trabajo, 46, 1-33.

Sandoval, E. (2008). Estudios sobre pobreza, marginación y desigualdad en Monterrey. Papeles de Población, 14(57), 169-191. 
Secretaría de Turismo (Sectur). (2004). Turismo alternativo, una nueva forma de hacer turismo. México: Autor.

Sierra, N., Romero, A. y Zizumbo, L. (2012). Desarrollo regional, electrificación y reorganización socioespacial en Valle de Bravo, México. Pueblos y Fronteras, 7(13), 243-269. Recuperado de http://redalyc.org/articulo. oa?id $=90624811009$ [2015, 20 de julio] .

Tomio, M. y Badel, K. (2009). Desarrollo regional del turismo y sustentabilidad del territorio. Perspectivas en Turismo, 18(6), 672-690. Recuperado de http://www.redalyc.org/articulo.oa?id = 180713900003 [2015, 18 de septiembre].

Torre, O. de la (1992). El turismo. Fenómeno social. México: Fondo de Cultura Económica.

Vázquez-Barquero, A. (1988). Desarrollo local: Una estrategia de creación de empleo. Madrid: Pirámide.

Vázquez-Barquero, A. (2009). Desarrollo local, una estrategia para tiempos de crisis. Universitas Forum, 1(2), 1-11. Recuperado de http://www.foromundialadel.org/experiencias/doc/Desarrollo \% 20Local, \%20estrategia \%20en \%20tiempos\%20de\%20crisis.pdf [2015, 3 de mayo].

Virgen, C. (2014). Turismo y desarrollo sustentable. Un acercamiento al estudio del turismo. México: Asociación Mexicana de Centros de Enseñanza Superior en Turismo y Gastronomía/Universidad de Guadalajara/Universidade Federal do Paraná. Recuperado de http://amestur.org/wpcontent/uploads/2015/03/Turismo-y-desarrollo-sustentable_digital. pdf [2015, 18 de septiembre]. 
